\title{
On the reactive ion etching of $\mathrm{RbTiOPO}_{4}$
}

\author{
A. Choudhary ${ }^{1}$, J. Cugat ${ }^{2}$, K. Pradeesh ${ }^{1}$, R. Solé ${ }^{2}$, F. Díaz ${ }^{2}$, M. Aguiló ${ }^{2}$, H. M. H. Chong ${ }^{3}$ and D. P. \\ Shepherd $^{1}$ \\ 1. Optoelectronics Research Centre, University of Southampton, Southampton, SO171BJ, United Kingdom \\ 2. Física i Cristal-lografia de Materials i Nanomaterials, Universitat Rovira i Virgili, Tarragona 43007, Spain \\ 3. Electronics and Computer Science, University of Southampton, Southampton, SO171BJ, United Kingdom
}

$\mathrm{RbTiOPO}_{4}$ (RTP) belongs to the $\mathrm{KTiOPO}_{4}(\mathrm{KTP})$ family of crystals and has similarly useful nonlinear optical properties. In addition, RTP allows higher doping of rare-earth elements, such as $\mathrm{Yb}^{3+}$, when compared to KTP, a 2 times larger damage threshold than KTP, a very large electro-optic coefficient, a low dielectric constant and high chemical stability, which makes these materials very interesting for active photonics applications. In particular, $\mathrm{Yb}^{3+}$-doped RTP exhibits a broad fluorescence spectrum that has been exploited to demonstrate ultrafast operation, with pulses as short as 155 fs being generated [1]. Recently, planar waveguiding has been demonstrated in an $\left(\mathrm{Yb}^{3+}, \mathrm{Nb}^{5+}\right)$ :RTP film grown by liquid phase epitaxy [2], opening the possibility of planar integrated devices based on this highly functional material.

Channel waveguides based on this thin-film technology would be of considerable interest for realising, for example, compact ultrafast lasers with GHz repetition rates [3] with applications in optical frequency metrology, optical sampling and biological imaging, or self-frequency-doubled lasers for visible laser sources. In this work we discuss the reactive ion etching (RIE) of RTP and the process optimisation to fabricate single-mode channel waveguides for lasing application around 1 micron.

A 200-nm layer of chromium (Cr) was deposited on a number of RTP substrates grown by the top-seeded solution growth method (TSSG), after which it was photolithographically patterned to give a Cr mask with waveguide features of widths $1 \mu \mathrm{m}$ to $10 \mu \mathrm{m}$. The masked substrates were etched in an OPT Plasmalab 80 plus RIE system (Oxford Instruments) with an RF frequency of $13.56 \mathrm{MHz}$. SF 6 and Ar were used to etch the substrates in order to study the interplay between chemical and physical etching processes, respectively. The gas flow rates, gas pressures and RF power were systematically varied to get a good balance between etch rate and RMS surface roughness. The dependence of the etch rate and surface roughness on each parameter is shown in figure 1. Based on these graphs the optimum conditions were chosen to be: RF power of $250 \mathrm{~W}$, gas pressure of $40 \mathrm{mTorr}$ and gas flow rate of $10 \mathrm{sccm}$ for both $\mathrm{SF}_{6}$ and Ar (giving a total gas flow rate of $20 \mathrm{sccm}$ ).

A 6- $\mu$ m-thick (Yb,Nb): RTP thin-film was grown on an undoped RTP substrate by liquid phase epitaxy and then etched for 135 minutes with the optimised conditions. It was then end polished to give a 6-mm-long waveguide. An SEM image of the end face of a 9.8- $\mu \mathrm{m}$-wide channel is shown in the inset of figure 1 . The sidewall angle was measured to be $63^{\circ}$ and the etch depth was $1.4 \mu \mathrm{m}$.

Waveguiding experiments were carried out by end-fire coupling a $981 \mathrm{~nm}$ fiber-coupled single-mode laser diode into the channels. The output was found to be single-mode, with $1 / \mathrm{e}^{2}$ mode diameters measured to be 16.4 $\mu \mathrm{m}$ and $8 \mu \mathrm{m}$ in the $\mathrm{x}$ and $\mathrm{y}$ directions respectively and is in good agreement with the simulated values of 15.2 $\mu \mathrm{m}$ and $6.8 \mu \mathrm{m}$.

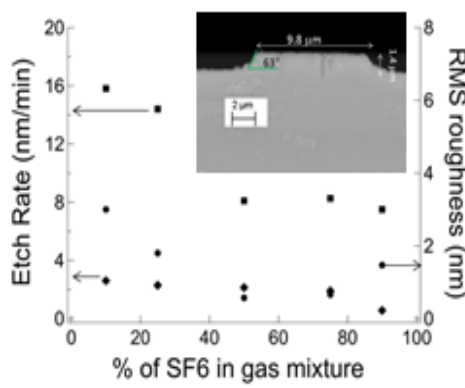

a.

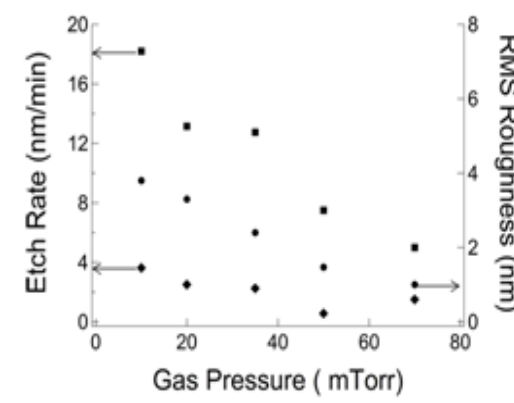

b.

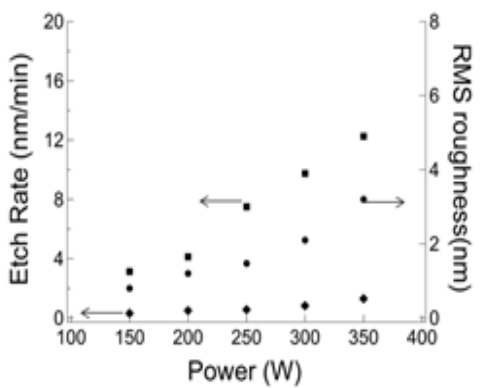

c.

Fig. 1 Dependence of etch rate and RMS surface roughness on a. percentage of $\mathrm{SF}_{6}$ in the $\mathrm{SF}_{6}$ : Ar gas mixture b. Gas pressure and c. the RF power. Squares- RTP etch rate, diamonds-Cr etch rate, circles- surface roughness. Inset in Fig.1a: SEM image of the cross-section of a $9.8 \mu \mathrm{m}$ waveguide.

\section{References}

[1] J. Carvajal, G. Ciatto, X. Mateos, A. Schmidt, U. Griebner, V. Petrov, G. Boulon, A. Brenier, A. Peña, M. Pujol, M. Aguiló, and F. Díaz, "Broad emission band of $\mathrm{Yb}^{3+}$ in the nonlinear Nb:RbTiOPO 4 crystal: origin and applications," Opt. Express , 18, $7228-7242$ (2010)

[2] J. Cugat, R. Solé, M.C. Pujol, J.J. Carvajal, X. Mateos, F. Díaz and M. Aguiló, "Waveguiding demonstration on Yb:Nb:RbTiOPO $4 / \mathrm{RbTiOPO}_{4}$ (001) epitaxies grown by LPE”, Opt. Mat., 32, 1648-1651 (2010)

[3] A.Choudhary, A.A.Lagatsky, K.Pradeesh, W.Sibbett, C.T.A.Brown, and D.P.Shepherd, "Diode-pumped femtosecond solid-state waveguide laser with a $4.9 \mathrm{GHz}$ pulse repetition rate”, Opt. lett., 37, 4416-4418 (2012) 